\title{
Clinical significance of leukocyte-associated immunoglobulin-like receptor-1 expression in human cervical cancer
}

\author{
YUE WANG, XUESHAN ZHANG, FANG MIAO, YANNING CAO, \\ JIANGNAN XUE, QIZHI CAO and XIAOSHU ZHANG
}

Department of Immunology, School of Basic Medicine, Binzhou Medical University, Yantai, Shandong 264003, P.R. China

Received June 17, 2015; Accepted October 10, 2016

DOI: $10.3892 /$ etm.2016.3842

\begin{abstract}
Leukocyte-associated immunoglobulin-like receptor-1 (LAIR-1) is broadly expressed on the majority of immune cells; however, the biological role of LAIR in solid tumors has yet to be elucidated. In the present study, using immunohistochemical staining analysis, the expression of LAIR-1 in human cervical cancer (HCC) and nontumor-adjacent tissue specimens was determined, and the results indicated that the expression of LAIR-1 in HCC tissue was higher compared with that in noncancerous tissue. The $\chi^{2}$ test was used to analyze the correlation between the expression of LAIR-1 in tumor tissues with clinicopathological parameters. The results showed that the expression of LAIR-1 in the cancer cell nucleus was significantly associated with tumor size, pathological differentiation, $\mathrm{T}$ classification and clinical stage. In addition, the expression in the cytoplasm was evidently associated with the number of positive lymph nodes. The HCC cell line, ME-180, which does not express LAIR-1, was stably transfected using LAIR-1 cDNA. Cell Counting Kit-8 and an annexin V assay showed that the overexpression of LAIR-1 in ME-180 cells suppressed the proliferation and anti-apoptosis capacity of the cells. These findings demonstrated that LAIR-1 is markedly overexpressed in HCC tissue, and that its expression status is associated with tumor progression. LAIR-1 may be a biomarker and target in the diagnosis and treatment of patients with HCC.
\end{abstract}

\section{Introduction}

Human cervical cancer (HCC) is the fourth most frequently diagnosed cancer and the fourth leading cause of cancer-related mortality in women (1). An estimated 52.8 million new cases

Correspondence to: Professor Xiaoshu Zhang, Department of Immunology, School of Basic Medicine, Binzhou Medical University, 346 Guanhai Road, Yantai, Shandong 264003, P.R. China E-mail: xiaoshu_zhang2006@163.com

Key words: human cervical cancer, leukocyte-associated immunoglobulin-like receptor-1, biomarker, inhibitory receptor and 26.6 million cases of mortality as a result of the disease were recorded in 2012 (2). However, the development of a widespread program for cervical cytologic screening (termed the Papanicolaou test) has substantially reduced HCC mortality (3). The majority ( $84.3 \%$ ) of cases occur in resource-poor countries, where the disease accounts for $\sim 12 \%$ of all cancers in women, and has an overall 5-year survival rate of $\sim 72 \%$ (2) The greatest risk factor for $\mathrm{HCC}$ is infection with certain types of the human papillomavirus (HPV), followed by smoking (4). The primary subtypes of HCC are squamous cell carcinoma and adenocarcinoma, and squamous cell carcinoma is the most commonly diagnosed subtype (5). The International Federation of Gynecology and Obstetrics (FIGO) staging system is used to determine the prognosis for patients, and provides the basis for therapeutic decision making (5). However, only $1 \%$ of women who test positive for HPV develop HCC (6). Therefore, it is important to identify a sensitive and specific cancer-related molecule that will serve as a predictive biomarker for clarifying the underlying molecular mechanisms of HCC, in addition to being a valuable diagnostic and therapeutic target.

The leukocyte-associated immunoglobulin-like receptor-1 (LAIR-1) is a member of the immunoglobulin (Ig) superfamily, and is a type I transmembrane glycoprotein. LAIR-1 is composed of a C2-type Ig-like domain and two immunoreceptor tyrosine-based inhibition motifs (ITIMs). It is broadly expressed on nearly all immune cells, such as T-cells, B-cells, and NK cells; however, it is not expressed on erythrocytes and platelets (7). It is also expressed on megakaryocytes (MKs) (8) and osteoclasts (9). Poggi et al (10) demonstrated that LAIR-1 was not expressed in high-risk B-cell chronic lymphocytic leukemia (CLL), and the intensity of expression in low- and intermediate-risk CLL was associated with the CLL stage and progression of the disease. However, a limited amount of attention has focused on LAIR-1 expression in solid tumors (11). The extracellular matrix (ECM) has been determined to promote the progression of a solid tumor (12). Collagen, an important component of ECM, has been confirmed to be the high-affinity ligand for LAIR-1 (13). Accordingly, LAIR-1 may be involved in tumor development.

The present study reports that LAIR-1 was markedly expressed in HCC specimens, and it was identified that 
overexpression of LAIR-1 was associated with clinicopathological features.

Thus, the present study found that the overexpression of LAIR-1 reduced tumor proliferation and increased tumor apoptosis of cervical carcinoma cells. These data suggest that HCC patients with a high level of LAIR-1 expression may not experience disease progression. This suggests that LAIR-1 may be an effective therapeutic biomarker and an alternative therapeutic target.

\section{Materials and methods}

Patients and tissue specimens. Fresh HCC and normal adjacent tissues were collected from 124 patients confirmed to have HCC between 2010 and 2012 at the Department of Gynecology, Affiliated Hospital of Binzhou Medical University (Yantai, China). All patients had squamous cell carcinoma tumors. No patients received radiotherapy, chemotherapy or immunotherapy prior to surgery. Patient ages ranged between 26 and 73 years (mean age, $44.8 \pm 10.4$ years). Tumor differentiation was assessed according to the FIGO staging system (5). Ethical approval was obtained from the Ethics Committee of Binzhou Medical University. Informed consent was obtained from all patients.

Immunohistochemical (IHC) staining. IHC staining was conducted to examine the altered expression of LAIR-1 in tumors and normal tissues. Formalin-fixed and paraffin-embedded sections were deparaffinized and rehydrated. Antigen retrieval was performed in a microwave at $98^{\circ} \mathrm{C}$ for $30 \mathrm{~min}$. The sections were treated with $3 \%$ hydrogen peroxide for $5 \mathrm{~min}$, and then blocked with $1 \%$ bovine serum albumin (AR1006; Boster Biological Technology,Ltd., Wuhan, China) for $30 \mathrm{~min}$. The sides were incubated with monoclonal mouse anti-human LAIR-1 (ab14826; Abcam, Cambridge, UK) at 1:500 dilution at $4^{\circ} \mathrm{C}$ overnight. Mouse IgG (BA1046; Boster Biological Technology, Ltd.) served as the negative control. The biotinylated anti-mouse secondary antibody (BA1001; Boster Biological Technology, Ltd.) was incubated the following day with the sides for $20 \mathrm{~min}$ at room temperature, followed by further treatment with a streptavidin-alkaline phosphatase complex (Boster Biological Technology, Ltd.) for $20 \mathrm{~min}$. The signal was detected using the BCIP/NBT substrate solution (Boster Biological Technology, Ltd.).

Semiquantitative assessment of IHC staining. The expression of LAIR-1 in the nucleus and cytoplasm was reviewed and scored by two independent observers who had no prior knowledge of the clinical information. The immunoreactivity was evaluated using a semiquantitative scoring system in which $0=$ no staining, $1=$ weak staining, $2=$ moderate staining, and $3=$ intense staining. The percentage of LAIR-1 positive cells scored as 0 was $0 \%$ (no positive cells), 1 was $\leq 25 \%, 2$ was between $25 \%$ and $50 \%, 3$ was between $50 \%$ and $75 \%$, and 4 was $>75 \%$. The immunoreactive score (IRS) was calculated as follows: Proportion of positive cells score $x$ intensity score. Values of IRS ranging between 0 and 12 were classified as follows: - (IRS: 0), + (IRS: 1-4), and ++ (IRS: 6-12).

Cell culture. Cervical squamous carcinoma cell lines (Ca Ski, ME-180, C-33 A, SiHa and MS751) were purchased from the Cell Bank of Shanghai Institutes for Biological Sciences (Shanghai, China). ME-180 cells were cultivated in McCoy's 5A medium (Sigma-Aldrich; Merck Millipore, Darmstadt, Germany), and C-33 A, SiHa and MS751 cells were maintained in Minimum Essential Medium (Gibco; Thermo Fisher Scientific, Inc., Waltham, MA, USA). Ca Ski cells were cultured in RPMI-1640 (Hyclone; GE Healthcare Life Sciences, Logan, UT, USA) medium. Media were supplemented with $10 \%$ fetal bovine serum (Hyclone) and incubated at $37^{\circ} \mathrm{C}$ under $5 \% \mathrm{CO}_{2}$ and saturated moisture.

Stable transfection. Sequence-verified LAIR-1 cDNA was cloned into a GV218 lentivirus vector (GeneChem Co., Ltd., Shanghai, China), which codes the Enhanced Green Fluorescent Protein (EGFP) gene. The accuracy of the recombinant vector was verified using automated sequencing (Thermo Fisher Scientific Inc.). The 293T cells were cotransfected with GV218-LAIR-1 or GV218, and other vectors (pHelper 1.0 and 2.0; GeneChem Co., Ltd.), and the viruses were subsequently harvested after $48 \mathrm{~h}$. The lentivirus was transfected into ME-180 cells, and cells transfected with an empty vector served as the negative control. At $48 \mathrm{~h}$ after transfection, the stable transfected cells, which were expressed as the vector sequences coding the puromycin resistance gene, were selected using puromycin (Sigma-Aldrich). The transfected cell lines were confirmed using western blotting and immunofluorescence.

Western blotting. Radioimmunoprecipitation assay lysis buffer (Beyotime Institute of Biotechnology, Nantong, China) was used to lyse the cells. Subsequent to centrifugation at $10,000 \mathrm{x} g$ for $15 \mathrm{~min}$ at $4^{\circ} \mathrm{C}$, the protein solution was collected and the concentration was quantitated using a BCA protein assay (Thermo Fisher Scientific Inc.). The protein (50 $\mu \mathrm{g}$ per lane) was separated using $10 \%$ sodium dodecyl sulfate-polyacrylamide gel electrophoresis and transferred to a polyvinylidene fluoride membrane. The membrane was probed with anti-LAIR-1 (ab14826; Abcam) at dilution of 1:500 and anti-GAPDH (AB-P-R 001; Goodhere Biotechnology Co., Ltd., Hangzhou, China) at dilution of $1: 10,000$ at $4^{\circ} \mathrm{C}$ overnight. After washing, the membrane was incubated with horseradish peroxidase-conjugated anti-rabbit/mouse secondary antibodies (1:2,500 dilution; BA1055/BA1051; Boster Biological Technology, Ltd.), at room temperature for $1.5 \mathrm{~h}$ and visualized using enhanced chemiluminescence plus (Millipore, Billerica, MA, USA).

Immunofluorescence staining. LAIR-1 transfected cells (L-ME-180) and cells transfected with empty vector (V-ME-180) were seeded into a cover glass at a density of $4 \times 10^{4} /$ piece $/ 500 \mu \mathrm{l}$ overnight. The following day, the cells were fixed with $4 \%$ paraformaldehyde for $1 \mathrm{~h}$ at $4^{\circ} \mathrm{C}$. After blocking, the cells were incubated with anti-LAIR- 1 at $4^{\circ} \mathrm{C}$ overnight prior to incubation with Cy3-conjugated anti-mouse IgG (ZDR-5210; ZSGB-BIO, Beijing, China) at a dilution of 1:80 for $1 \mathrm{~h}$ at room temperature. The cells were analyzed using laser confocal scanning microscopy (LEICA Microsystems GmbH, Wetzlar, Germany).

Cell proliferation assay. Cells were seeded into a 96-well plate at a concentration of 4,000/well/100 $\mu \mathrm{l}$. The cells were quantified at $0,24,48$, and $72 \mathrm{~h}$ using a Cell Counting Kit-8 (CCK-8) (Dojindo Co., Ltd., Kumamoto, Japan), and the absorbance 
Table I. Differential LAIR-1 expression in hec and matched normal tissues.

\begin{tabular}{|c|c|c|c|c|c|c|c|c|}
\hline \multirow[b]{2}{*}{ Tissue type } & \multicolumn{4}{|c|}{ Cytoplasmic LAIR-1 } & \multicolumn{4}{|c|}{ Nuclear LAIR-1 } \\
\hline & - & + & ++ & P-value & - & + & ++ & P-value \\
\hline HCC tissues & $15(12.1)$ & $54(43.5)$ & $55(44.4)$ & $<0.001$ & $61(49.2)$ & $49(39.5)$ & $14(11.3)$ & 0.026 \\
\hline Normal tissues & $113(91.1)$ & $11(8.9)$ & $0(0.0)$ & & $63(50.8)$ & $56(45.2)$ & $5(4.0)$ & \\
\hline
\end{tabular}

LAIR-1, leukocyte-associated immunoglobulin-like receptor-1.

was detected at $450 \mathrm{~nm}$. Cell proliferation viability was recorded according to the following equation: Cell proliferation viability $=$ absorbance value $(\mathrm{AV}) / 0 \mathrm{~h} \mathrm{AV}$. The experiment was performed in triplicate and repeated three times.

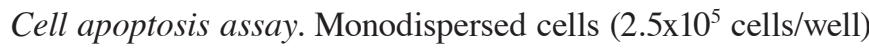
were seeded into a 6-well plate in the maintenance medium. Subsequent to incubation for $24 \mathrm{~h}$, cells were treated with $0.5 \mu \mathrm{g} / \mathrm{ml}$ cisplatin (Hansoh Pharmaceutical Co., Ltd., Lianyungang, China) for $18 \mathrm{~h}$, and then digested with $0.25 \%$ trypsin (ethylenediaminetetraacetic acid-free). The Annexin V-PE Apoptosis Detection kit (Keygen Biotechnology Co., Ltd., Nanjing, China) was used for staining. The cells were analyzed using a flow cytometer (BD Biosciences, Franklin Lakes, NJ, USA).

Statistical analysis. Data was analyzed using SPSS software (version 15.0; SPSS, Inc., Chicago, IL, USA). The Wilcoxon exact test was used to determine the LAIR-1 expression in $\mathrm{HCC}$ and adjacent nontumor cervical tissues. The association between LAIR-1 and various clinicopathological features was analyzed using the $\chi^{2}$ test. Continuous variables were analyzed using two-way analysis of variance. The correlation between the pathological stage and LAIR-1 expression levels were assessed using Spearman's Rank correlation coefficient analysis. $\mathrm{R}<0$ was considered to indicate negative correlation, and $\mathrm{P}<0.05$ was considered to indicate a statistically significant difference.

\section{Results}

LAIR-1 is upregulated in HCC clinical samples. To investigate the association between LAIR-1 expression and cervical lesions, HCC and adjacent normal cervical tissues were examined by IHC staining. Representative results are shown in Fig. 1. LAIR-1 was localized in the cytoplasm and nucleus of the cells, and was primarily expressed in the cytoplasm. The percentage of cells that were $>90 \%$ positive was $48.4 \%$ in the cytoplasm of HCC specimens (data not shown). As summarized in Table I, compared with normal adjacent tissues, LAIR-1 overexpression in HCC tissues was statistically significant both in the cytoplasm and nucleus $(\mathrm{P}<0.05)$. Of the $124 \mathrm{HCC}$ patient samples analyzed, positive staining of LAIR-1 in the cytoplasm was detected in 109 tumors (88\%); 54 cases exhibited weak LAIR-1 staining, and 55 cases showed strong LAIR-1 staining. By contrast, only 11 cases $(8.9 \%)$ exhibited weak staining in the cytoplasm of the paired tumor-adjacent normal tissues, and no cases showed strong staining. Although the negative rate of LAIR-1 expression in the nucleus of HCC tissue was $49.2 \%$, the negative rate in normal tissues was $50.8 \%$, and the difference was statistically significant $(\mathrm{P}=0.026)$.

LAIR-1 expression is correlated to clinicopathological variables. The correlation between LAIR-1 expression and clinicopathological parameters was analyzed using the $\chi^{2}$ test. Table II indicates that LAIR-1 expression in the cytoplasm was significantly associated with the number of positive lymph nodes $(\mathrm{P}<0.001)$; however, no statistically significant associations between LAIR-1 expression and age, tumor size, pathologic stage, $\mathrm{T}$ classification, $\mathrm{N}$ classification and clinical stage $(\mathrm{P}>0.05)$ were detected. In the nucleus, LAIR-1 expression was evidently associated with tumor size $(\mathrm{P}=0.047)$, pathological stage $(\mathrm{P}=0.014)$, $\mathrm{T}$ classification $(\mathrm{P}<0.001)$ and clinical stage $(\mathrm{P}=0.027)$. A negative correlation existed between LAIR-1 expression and the pathological stage $(\mathrm{R}=-0.185, \mathrm{P}=0.043)$.

LAIR-1 was overexpressed in ME-180 cell line. To research the functional significance of LAIR-1 in HCC, its expression was detected in HCC cell lines (Ca Ski, ME-180, C-33 A, SiHa, and MS751); however, no expression was detected (data not shown). The LAIR-1 expression levels were modulated in ME-180 cells by using stable transfection with the LAIR-1 expression plasmid. Western blotting and immunofluorescence analysis confirmed an increase of LAIR-1 expression in ME-180 cells after transfection. As hypothesized, we observed enhanced expression of LAIR-1 in the ME-180 cells (L-ME-180; Fig. 2A), and the molecular weight was $70 \mathrm{kDa}$ as a result of the fusion of LAIR-1 and EGFP proteins (Fig. 2B).

LAIR-1 expressed in HCC cell lines is tumor suppressive. To investigate the function of LAIR-1 in the growth of HCC cells, CCK-8 and annexin $\mathrm{V}$ assays were used to detect the proliferation and apoptosis of ME-180 cells. Cell proliferation ability was significantly reduced in the L-ME-180 cells ( $\mathrm{P}<0.001$; Fig. 3A). As shown in Fig. 3B, compared with V-ME-180 and ME-180 cells, respectively, the mean apoptotic rate of L-ME-180 cells increased $(\mathrm{P}=0.021$ or $\mathrm{P}=0.020)$. In summary, the expression of LAIR-1 inhibits the proliferation and promotes the apoptosis of ME-180 cells.

\section{Discussion}

In industrialized countries, $\mathrm{HCC}$ incidence has been significantly reduced as a result of organized screening programs (14). However, new cases of HCC in less developed regions without access to health care account for $84.3 \%$ of the worldwide incidence (1). High-risk HPV infection is an 
Table II. Correlation between human cervical cancer clinicopathological features with LAIR-1 expression in subcellular localization.

\begin{tabular}{|c|c|c|c|c|c|c|c|c|}
\hline \multirow[b]{2}{*}{ Variable } & \multicolumn{4}{|c|}{ Cytoplasmic LAIR-1 } & \multicolumn{4}{|c|}{ Nuclear LAIR-1 } \\
\hline & $\begin{array}{c}- \\
(\mathrm{n}=15)\end{array}$ & $\begin{array}{c}+ \\
(n=54)\end{array}$ & $\begin{array}{c}++ \\
(n=55)\end{array}$ & P-value & $\begin{array}{c}- \\
(n=61)\end{array}$ & $\begin{array}{c}+ \\
(n=49)\end{array}$ & $\begin{array}{c}++ \\
(n=14)\end{array}$ & P-value \\
\hline Age & & & & 0.756 & & & & 0.780 \\
\hline$\leq 45$ & $9(12.9)$ & $32(45.7)$ & $29(41.4)$ & & $33(47.1)$ & $28(40.0)$ & $9(12.9)$ & \\
\hline$>45$ & $6(11.1)$ & $22(40.7)$ & $26(48.1)$ & & $28(51.9)$ & $21(38.9)$ & $5(9.3)$ & \\
\hline Tumor size, cm & & & & 0.751 & & & & 0.047 \\
\hline$\leq 4$ & $12(13.5)$ & $38(42.7)$ & $39(43.8)$ & & $38(42.7)$ & $41(46.1)$ & $10(11.2)$ & \\
\hline$>4$ & $3(8.6)$ & $16(45.7)$ & $16(45.7)$ & & $23(65.7)$ & $8(22.9)$ & $4(11.4)$ & \\
\hline Pathological stage & & & & 0.390 & & & & 0.014 \\
\hline $\mathrm{G} 1$ & $0(0.0)$ & $3(42.9)$ & $4(57.1)$ & & $1(14.3)$ & $3(42.9)$ & $3(42.9)$ & \\
\hline $\mathrm{G} 2$ & $9(9.4)$ & $43(44.8)$ & $44(45.8)$ & & 45 (46.9) & $43(44.8)$ & $8(8.3)$ & \\
\hline G3 & $4(23.5)$ & $7(41.2)$ & $6(35.3)$ & & $11(64.7)$ & $3(17.6)$ & $3(17.6)$ & \\
\hline \multicolumn{9}{|l|}{ Number of } \\
\hline positive lymph nodes & & & & $<0.001$ & & & & 0.492 \\
\hline 0 & $9(10.7)$ & $37(44.0)$ & $38(45.2)$ & & $45(53.6)$ & $32(38.1)$ & $7(8.3)$ & \\
\hline$\leq 3$ & $0(0.0)$ & $7(36.8)$ & $12(63.2)$ & & $9(47.4)$ & $7(36.8)$ & $3(15.8)$ & \\
\hline$>3$ & $5(41.7)$ & $3(25.0)$ & $4(33.3)$ & & $5(41.7)$ & $4(33.3)$ & $3(25.0)$ & \\
\hline T classification & & & & 0.090 & & & & $<0.001$ \\
\hline $\mathrm{T} 1$ & $12(15.0)$ & $37(46.3)$ & $31(38.8)$ & & $39(48.8)$ & $37(46.3)$ & $4(5.0)$ & \\
\hline $\mathrm{T} 2$ & $3(8.8)$ & $14(41.2)$ & $17(50.0)$ & & $21(61.8)$ & $9(26.5)$ & $4(11.8)$ & \\
\hline T3 & $0(0.0)$ & $0(0.0)$ & $5(100.0)$ & & $0(0.0)$ & $2(40.0)$ & $3(60.0)$ & \\
\hline $\mathrm{N}$ classification & & & & 0.746 & & & & 0.251 \\
\hline N0 & $9(10.8)$ & $37(44.6)$ & $37(44.6)$ & & $45(54.2)$ & $32(38.6)$ & $6(7.2)$ & \\
\hline N1 & $6(15.8)$ & $16(42.1)$ & $16(42.1)$ & & $16(42.1)$ & $16(42.1)$ & $6(15.8)$ & \\
\hline Clinical stage & & & & 0.914 & & & & 0.027 \\
\hline 1 & $6(12.8)$ & $22(46.8)$ & $19(40.4)$ & & $24(51.1)$ & $22(46.8)$ & $1(2.1)$ & \\
\hline 2 & $3(9.7)$ & 13 (41.9) & $15(48.4)$ & & $20(64.5)$ & $8(25.8)$ & $3(9.7)$ & \\
\hline 3 & $6(14.6)$ & $16(39.0)$ & $19(46.3)$ & & $16(39.0)$ & $17(41.5)$ & $8(19.5)$ & \\
\hline
\end{tabular}

LAIR-1, leukocyte-associated immunoglobulin-like receptor-1; -, immunoreactive score (IRS) 0; +, IRS 1-4; ++, IRS 6-12.

essential and causal factor for HCC. However, establishing a cytology-based program in low-resource countries has several limitations. Since 2000, a range of novel biomarkers has been identified to advance the current understanding of HCC molecular mechanisms, including viral biomarkers (HPV DNA detection, HPV integration, HPV oncogene mRNA and host and viral methylation) and cellular biomarkers ( $\mathrm{p} 16^{\text {ink4a }}$, MCM2, Top2a, ki-67, 3q, and 5p chromosomal instability) (15). Numerous researchers are currently investigating various biomarker candidates.

LAIR-1 is primarily expressed on immune cells, and is also presented on $\mathrm{CD}_{3} 4^{+}$hematopoietic progenitor cells (7), MKs (8) and osteoclasts (9). By using an IHC staining approach for analyzing LAIR-1 expression in primary untreated HCC and normal cervical epithelia, the current study observed LAIR-1 to be localized in the cytoplasm and nucleus of HCC cells. The strong expression of LAIR-1 in HCC tissues indicated that LAIR-1 may serve a role in HCC progression. According to the statistical analysis to elucidate the correlation between LAIR-1 expression and clinicopathological variables, it was confirmed that the level of LAIR-1 expression in the cytoplasm was associated with the number of positive lymph nodes in patients with HCC, and that LAIR-1 expression in the cell nucleus was associated with pathological stage, $\mathrm{T}$ classification and clinical stage. In conclusion, the expression levels of LAIR-1 were associated HCC progression.

The present study used the ME-180 HCC cell line to further investigate the function of elevated LAIR-1 expression in HCC tissues. LAIR-1 expression is not detected on common HCC cell lines. The current study established that LAIR-1 may be stably transfected into ME-180 cells. Previous studies have demonstrated the inhibitory function of LAIR-1 (16-18). Poggi et al (19) stated that LAIR-1 is able to inhibit proliferation and induce apoptosis of the myelomonocytic THP1, MM6 and U937 cell lines through a caspase-independent pathway. LAIR-1 engagement may also downregulate granulocyte-macrophage 

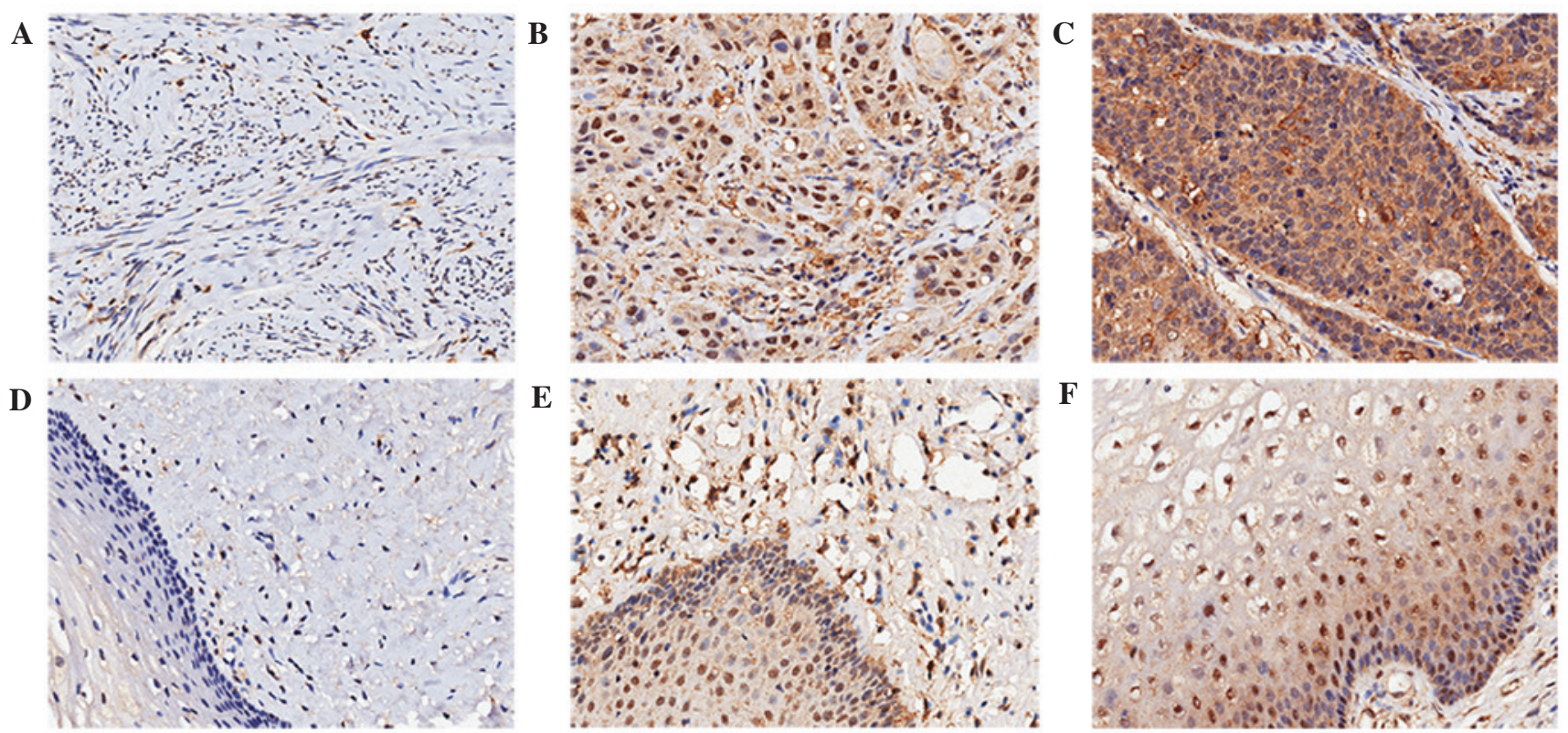

Figure 1. LAIR-1 expression in HCC and nontumor adjacent tissues. IHC staining was used to detect LAIR-1 expression in tissue specimens. HCC tissues with (A) negative (B) weak, and (C) strong LAIR-1 staining. Nontumor adjacent tissues with (D) negative (E) weak, and (F) strong LAIR-1 staining. Original magnification, x200. LAIR-1, leukocyte-associated immunoglobulin-like receptor-1; IHC, immunohistochemical; HCC, human cervical cancer.

A

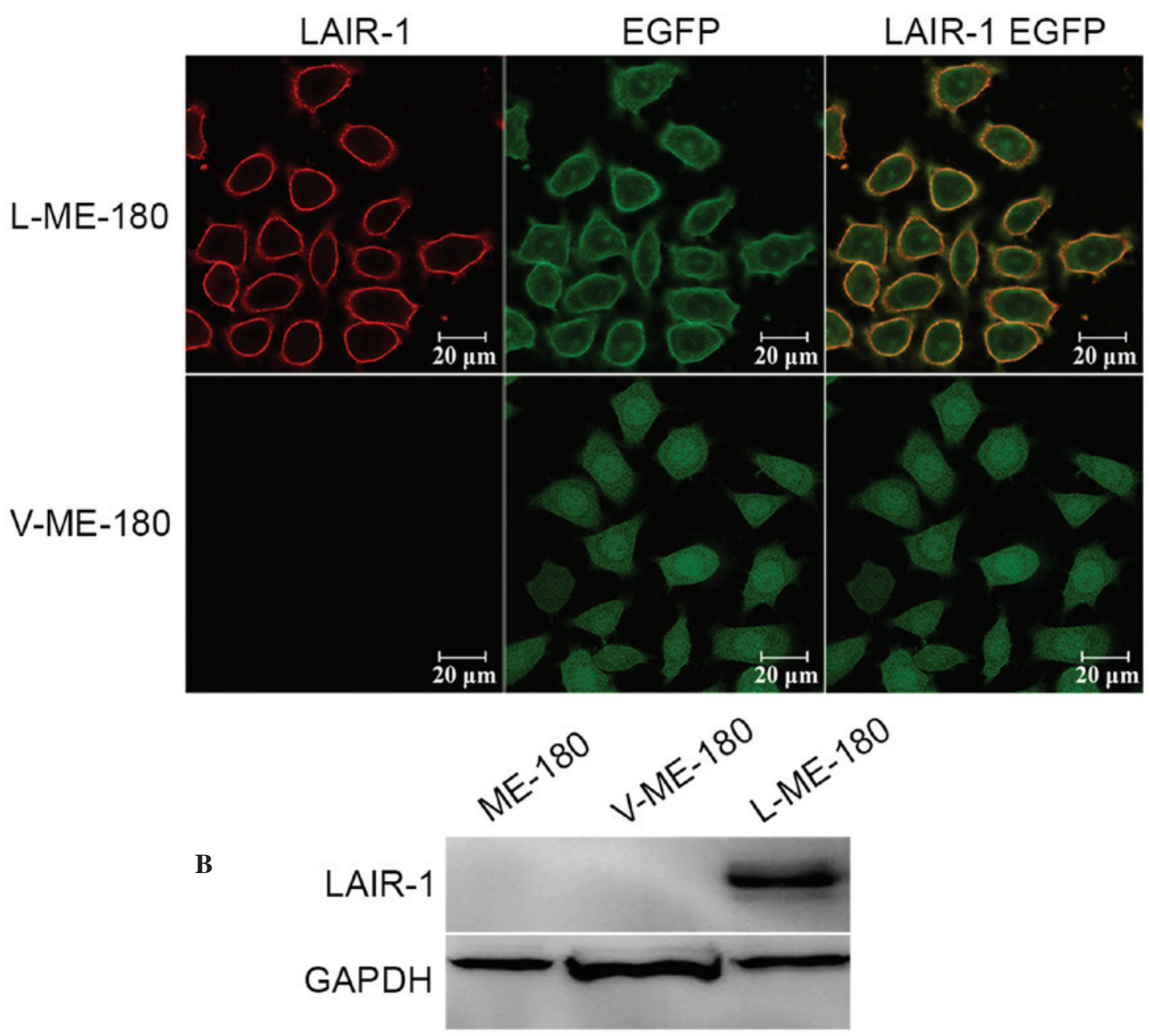

Figure 2. LAIR-1 expression on ME-180, L-ME-180 and V-ME-180 cells. (A) Laser confocal scanning microscopy was used to analyze LAIR-1 expression. Cells were incubated with Cy3-conjugated anti-LAIR-1 mAb (red). EGFP (green) was expressed in stably transfected cells (L-ME-180) and control cells (V-ME-180). The colocalization of LAIR-1 and EGFP is shown (yellow). (B) Western blotting was used to detect LAIR-1 expression in these cells. GAPDH was used as an internal control. LAIR-1, leukocyte-associated immunoglobulin-like receptor-1; EGFP, enhanced green fluorescent protein. 
A

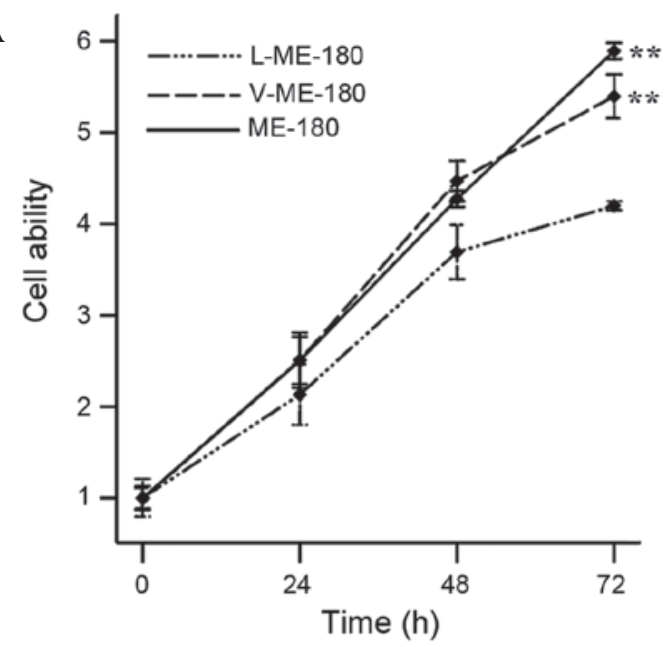

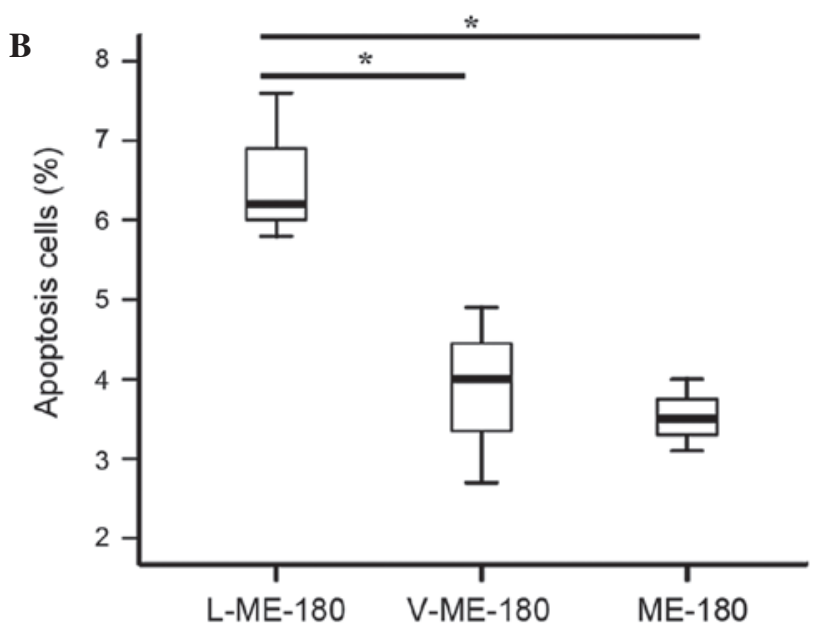
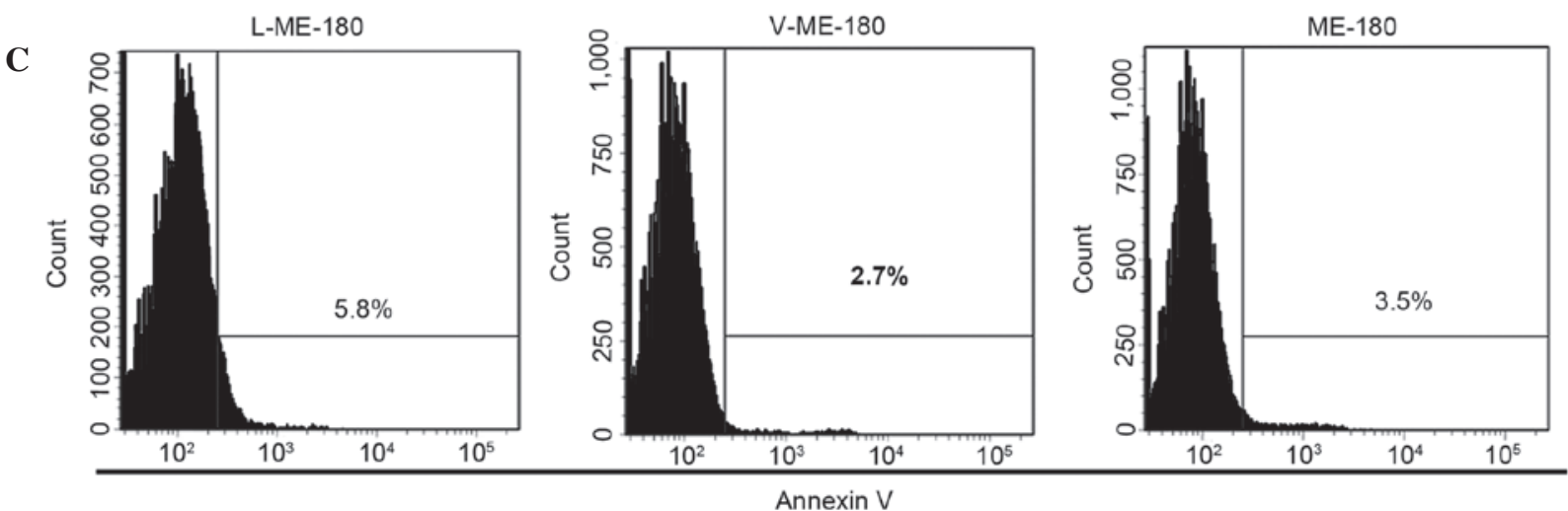

Figure 3. LAIR-1 can reduce the proliferation and induce the apoptosis of ME-180 cells. (A) A CCK-8 assay was used to assess the proliferation of L-ME-180, V-ME-180 and ME-180 cells. ${ }^{*}$ P $<0.001$ vs. L-ME-180 at $72 \mathrm{~h}$. (B) The apoptosis rates of ME-180 cells after LAIR-1 transfection were determined using an annexin $\mathrm{V}$ assay. Three independent experiments determined the percentage of the apoptotic rates. ${ }^{*} \mathrm{P}<0.05$. (C) The rate of cell apoptosis was detected using flow cytometry. The results of one experiment show that the difference between L-ME-180 and V-ME-180 (or parental ME-180) was statistically significant. Comparisons were conducted using ANOVA. LAIR-1, leukocyte-associated immunoglobulin-like receptor-1.

colony-stimulating factor-mediated survival and proliferation of acute myeloid leukemia blasts (20). The present study demonstrated that LAIR-1 expression suppresses the ability of ME-180 cells to proliferate and induces their apoptosis. The two ITIMs of LAIR-1 are commonly considered as the basis for inhibitory function. Phosphatizing the tyrosine residues of ITIMs can recruit the effectors of Src homology phosphotyrosine phosphatase-1 (SHP-1) and SHP-2, and send an intracellular signal. SHP-1 has been reported to be a negative regulator of angiogenesis (21), and is overexpressed in breast cancer (22). In addition, upregulation of SHP-2 has been found in gastric cancer $(23)$ and HCC $(24,25)$. Cells or tumor stroma can produce collagen (12), and cross-linking LAIR-1 with collagen can phosphatize ITIMs, recruit SHP-1 and SHP-2 (26), and weaken activation signals. Future studies will focus on the role of LAIR-1 in the growth of HCC cells, and the underlying mechanisms.

In conclusion, high expression of LAIR-1 in HCC is associated with clinicopathological variables and inhibits the growth ability of HCC cell lines. The present results suggests that LAIR-1 may be a novel biomarker for diagnosis and a treatment target for patients with HCC.

\section{Acknowledgements}

The present study was supported by the National Natural Science Foundation of China (grant no. 81001300), the Shandong Provincial Natural Science Foundation of China (grant no. ZR2010HM072) and the Scientific Research Development Program of Shandong Provincial Education Department of China (grant no. J08LG04).

\section{References}

1. Torre LA, Bray F, Siegel RL, Ferlay J, Lortet-Tieulent J and Jemal A: Global Cancer Statistics, 2012. CA Cancer J Clin 65: 87-108, 2015.

2. Ferlay J, Soerjomataram I, Dikshit R, Eser S, Mathers C, Rebelo M, Parkin DM, Forman D and Bray F: Cancer incidence and mortality worldwide: Sources, methods and major patterns in GLOBOCAN 2012. Int J Cancer 136: E359-E386, 2015.

3. Dhillon PK, Yeole BB, Dikshit R, Kurkure AP and Bray F: Trends in breast, ovarian and cervical cancer incidence in Mumbai, India over a 30-year period, 1976-2005: An age-period-cohort analysis. Br J Cancer 105: 723-730, 2011.

4. Gadducci A, Barsotti C, Cosio S, Domenici L and Riccardo Genazzani A: Smoking habit, immune suppression, oral contraceptive use, and hormone replacement therapy use and cervical carcinogenesis: A review of the literature. Gynecol Endocrinol 27: 597-604, 2011. 
5. Benedet JL, Bender H, Jones H III, Ngan HY and Pecorelli S: FIGO staging classifications and clinical practice guidelines in the management of gynecologic cancers. FIGO Committee on Gynecologic Oncology. Int J Gynaecol Obstet 70: 209-262, 2000.

6. Cain JM and Howett MK: Preventing cervical cancer. Science 288 : 1753-1755, 2000.

7. Meyaard L: LAIR and collagens in immune regulation. Immuno Lett 128: 26-28, 2010

8. Xue J, Zhang X, Zhao H, Fu Q, Cao Y, Wang Y, Feng X and Fu A: Leukocyte-associated immunoglobulin-like receptor-1 is expressed on human megakaryocytes and negatively regulates the maturation of primary megakaryocytic progenitors and cell line Biochem Biophys Res Commun 405: 128-133, 2011.

9. Zhang Y, Ding Y, Huang Y, Zhang C, Boquan J and Ran Z: Expression of leukocyte-associated immunoglobulin-like receptor-1 (LAIR-1) on osteoclasts and its potential role in rheumatoid arthritis. Clinics (Sao Paulo) 68: 475-481, 2013.

10. Poggi A, Catellani S, Bruzzone A, Caligaris-Cappio F, Gobbi M and Zocchi MR: Lack of the leukocyte-associated Ig-like receptor-1 expression in high-risk chronic lymphocytic leukaemia results in the absence of a negative signal regulating kinase activation and cell division. Leukemia 22: 980-988, 2008.

11. Cao Q, Fu A, Yang S, He X, Wang Y, Zhang X, Zhou J, Luan X, $\mathrm{Yu} \mathrm{W}$ and Xue J: Leukocyte-associated immunoglobulin-like receptor-1 expressed in epithelial ovarian cancer cells and involved in cell proliferation and invasion. Biochem Biophys Res Commun 458: 399-404, 2015.

12. Lu P, Weaver VM and Werb Z: The extracellular matrix: A dynamic niche in cancer progression. J Cell Biol 196: 395-406, 2012.

13. Lebbink RJ, de Ruiter T, Adelmeijer J, Brenkman AB, van Helvoort JM, Koch M, Farndale RW, Lisman T, Sonnenberg A, Lenting PJ and Meyaard L: Collagens are functional, high affinity ligands for the inhibitory immune receptor LAIR-1. J Exp Med 203: 1419-1425, 2006.

14. Tewari KS, Sill MW, Long HJ 3rd, Penson RT, Huang H, Ramondetta LM, Landrum LM, Oaknin A, Reid TJ, Leitao MM Michael HE and Monk BJ: Improved survival with Bevacizumab in advanced cervical cancer. N Engl J Med 370: 734-743, 2014.

15. Sahasrabuddhe VV, Luhn $P$ and Wentzensen N: Human papillomavirus and cervical cancer: Biomarkers for improved prevention efforts. Future Microbiol 6: 1083-1098, 2011.

16. Meyaard L, Adema GJ, Chang C, Woollatt E, Sutherland GR, Lanier LL and Phillips JH: LAIR-1, a novel inhibitory receptor expressed on human mononuclear leukocytes. Immunity 7 : 283-290, 1997.
17. Ouyang W, Ma D, Lin D, Sun Y, Liu X, Li Q, Jia W, Cao Y, Zhu Y and Jin B: 9.1C3 is identical to LAIR-1, which is expressed on hematopoietic progenitors. Biochem Biophys Res Commun 310: 1236-1240, 2003

18. Maasho K, Masilamani M, Valas R, Basu S, Coligan JE and Borrego F: The inhibitory leukocyte-associated Ig-like receptor-1 (LAIR-1) is expressed at high levels by human naive T cells and inhibits TCR mediated activation. Mol Immunol 42: 1521-1530, 2005.

19. Poggi A, Pellegatta F, Leone BE, Moretta L and Zocchi MR: Engagement of the leukocyte-associated Ig-like receptor-1 induces programmed cell death and prevents NF-kappaB nuclear translocation in human myeloid leukemias. Eur J Immunol 30: 2751-2758, 2000.

20. Zocchi MR, Pellegatta F, Pierri I, Gobbi M and Poggi A: Leukocyte-associated Ig-like receptor-1 prevents granulocyte-monocyte colony stimulating factor-dependent proliferation and Akt1/PKB alpha activation in primary acute myeloid leukemia cells. Eur J Immunol 31: 3667-3675, 2001.

21. Seo DW, Li H, Qu CK, Oh J, Kim YS, Diaz T, Wei B, Han JW and Stetler-Stevenson WG: Shp-1 mediates the antiproliferative activity of tissue inhibitor of metalloproteinase-2 in human microvascular endothelial cells. J Biol Chem 281: 3711-3721, 2006.

22. Yip SS, Crew AJ, Gee JM, Hui R, Blamey RW, Robertson JF, Nicholson RI, Sutherland RL and Daly RJ: Up-regulation of the protein tyrosine phosphatase SHP-1 in human breast cancer and correlation with GRB2 expression. Int J Cancer 88: 363-368, 2000.

23. Jiang J, Jin MS, Kong F, Wang YP, Jia ZF, Cao DH, Ma HX, Suo J and Cao XY: Increased expression of tyrosine phosphatase SHP-2 in Helicobacter pylori-infected gastric cancer. World J Gastroenterol 19: 575-580, 2013

24. Meng F, Zhao X and Zhang S: Expression and significance of SHP-2 in human papillomavirus infected cervical cancer. J Huazhong Univ Sci Technolog Med Sci 32: 247-251, 2012.

25. Tao XH, Shen JG, Pan WL, Dong YE, Meng Q, Honn KV and Jin R: Significance of SHP-1 and SHP-2 expression in human papillomavirus infected Condyloma acuminatum and cervical cancer. Pathol Oncol Res 14: 365-371, 2008.

26. Verbrugge A, Rijkers ES, de Ruiter T and Meyaard L: Leukocyte-associated Ig-like receptor-1 has $\mathrm{SH} 2$ domain-containing phosphatase-independent function and recruits C-terminal Src kinase. Eur J Immunol 36: 190-198, 2006. 